\title{
Moving Object Databases - Dynamic Flight Route Modification According to Weather Related Incidents
}

\author{
Konuralp Küçüködük*, Perihan Pehlivanoğlu \\ Turkish Air Force Academy, Computer Engineering Department, İstanbul, Turkey. \\ * Corresponding author. Tel.: +905534527919; email: konuralp-k@hvkk.tsk.tr \\ Manuscript submitted February 17, 2015; accepted July 10, 2015. \\ doi: 10.17706/ijcee.2015.7.4.232-239
}

\begin{abstract}
A moving entity requires huge amount of data, thus its database should handle these data (index mechanisms, secondary storage needs, its management of data for multiusers, and so on). To give an object's correct location, database need to be updated frequently. This means capacity gets overflow eventually. So, new data types need to be used to make the system stable. The solution is to unite spatial and temporal data. It's called spatio-temporal data. Also, this type of database is called moving objects databases (MODS). With these type of data it is possible to show plane locations and dynamically change air vehicle's (UAV, plane, military vehicle and so on) route according to weather constantly. In this study Airborne Warning and Control System (AWACS) movement is modeled, this air vehicle's data is queried, and dynamically its route calculated according to weather. Therefore, a safer route can be calculated and planned in today's technologic capabilities.
\end{abstract}

Key words: Moving object databases, secondo, spatio-temporal data, dynamic route planning.

\section{Introduction}

Usage of moving object data increases all the time with the advances at satellite, Global Positioning System (GPS) and wireless sensor network technology. GPS technology promotes many new applications and uses moving object databases at various fields. Animal scientists track wildlife to investigate environmental act; merchants insert GPS in cars to monitor and guide vehicles; meteorologists use weather satellites to observe weather condition and Unmanned Aerial Vehicles track the movement of terrorists [1]. Moving entities could be GPS attached electrical equipment: such as planes, ships, human beings and animals. These are called moving points. Still, there are some other moving entities called moving regions. For example; snow, rain, a group of terrorists, migrating birds or ashes comes out of a volcanic eruption. The primary target of this research is to see the movement of Airborne Warning and Control System (AWACS) and secondly querying the objects at weather and earth surface which affect AWACS. Lastly, simulate the queries.

The research is simulated at a prototype database system called Secondo. This system is able to demonstrate moving objects, continuously changing time-dependent geometries. Secondo has capability to represent complete histories of movement. Usage of the system is very practical. Previously, it's used at academic studies at all over the world.

Lately, a research done at Milan and Fern University, Secondo is used to illustrate hybrid trajectories. Researchers combined the symbolic and geometric dimensions in a multi-dimensional trajectory. Symbolic trajectories can be used to represent the activities performed by an individual in time, based on the moving object data model. A symbolic trajectory is defined by an object of type moving label. Temporarily 
interrelated trajectories, describing the movement with different ways complicates the analysis of moving data. For example; the pattern of transportation starts with taxi then if it's followed by bus, some classification need to be done. Multi-dimensional trajectory enables integration of the classical moving object data model with the symbolic data model [2].

At a study done last year at Turkish Air Force Academy, unmanned aerial vehicle (UAV) routes are simulated and indexing algorithms have been used to analyze UAV data. Demonstration was done with a prototype database system called Secondo. UAV is an aircraft which is under remote or autonomous control mostly used for civilian or military complex missions has high technology in all related systems. In military concept UAV is used for various purposes like dangerous, reconnaissance, multi collaborative missions, etc. The study has done by using R-Tree and TB-Tree indexing algorithms to find the nearest neighbor method. Nearest neighbor method, finds two nearest objects of the selected object. This method prevents unnecessary processes and minimizes disc accesses to make efficient and fast querying possible. Indexing structure is directly proportional with the performance efficiency [3].

At our research, to show moving entities, we used the same prototype database system, Secondo. We created moving points which represent AWACS and moving regions which represent clouds. AWACS have planned departure time, specific routes and missions. Normally if a cloud or thunderstorm approaches from Europe or Asia the meteorologists observe the movement of weather and inform the flight crew before flight. With Secondo, movement of weather can be shown at graphical user interface (GUI) and new flight route can be planned or departure time can be changed to avoid from meteorological conditions. Apart from AWACS squadron, this type of system can be used at every Turkish Air Force squadrons. Flight safety is a critical subject. Thus, by using this system, flight safety can be highly maintained.

The rest of the paper is organized as follows. Section 2 explains characteristics of moving object database parts. Section 3 gives brief information about Secondo database system. Section 4 expresses the AWACS model, affection of weather condition over flights and benefits of the system. Section 5 concludes the paper.

\section{Moving Object Databases}

\subsection{Design}

The necessity of moving object databases (MOD) is that classical database management systems (DBMS's) are not applicable enough to demonstrate constantly changing data. Traditional databases replies only if it is updated, thus they only handle past movements of moving objects. For example; AWACS was above Ankara at 10:00 am and above Kayseri at 10:15 am. The coordinates updated only at that time. Traditional databases should not support the query such as; where the location of AWACS at 10:09 was. To observe the correct location of AWACS, database supposed to be updated very often but this is not feasible. This situation requires very high capacity and getting back-up is difficult. Even if system updates frequently, it is possible to have disconnections. Another important problem is loss of performance, systems slows down, and for a military mission this causes inconsistencies and instabilities.

\subsection{Spatial Databases}

The purpose of spatial databases is to change data structures to be capable to query geometric shapes and algorithms. For modelling objects, there are three main types; point, line and region. [4] A point represents an object's location in space. Examples of point objects are airports, cities. A line is the basic type for moving through space or junctions in space. Examples of line objects are a route of a plane, a borderline of a country. Lastly, a region is the type for an entity having an area in the 2D space. [4] Examples of region objects are countries, clouds, group of terrorists or lakes. The three basic types are illustrated at Fig. 1. Red line is AWACS04s trajectory, red point is Antalya Airbase, green point is AWACS04 and grey region is a cloud. Fig. 1 shows a mission over Antalya, Turkey. 


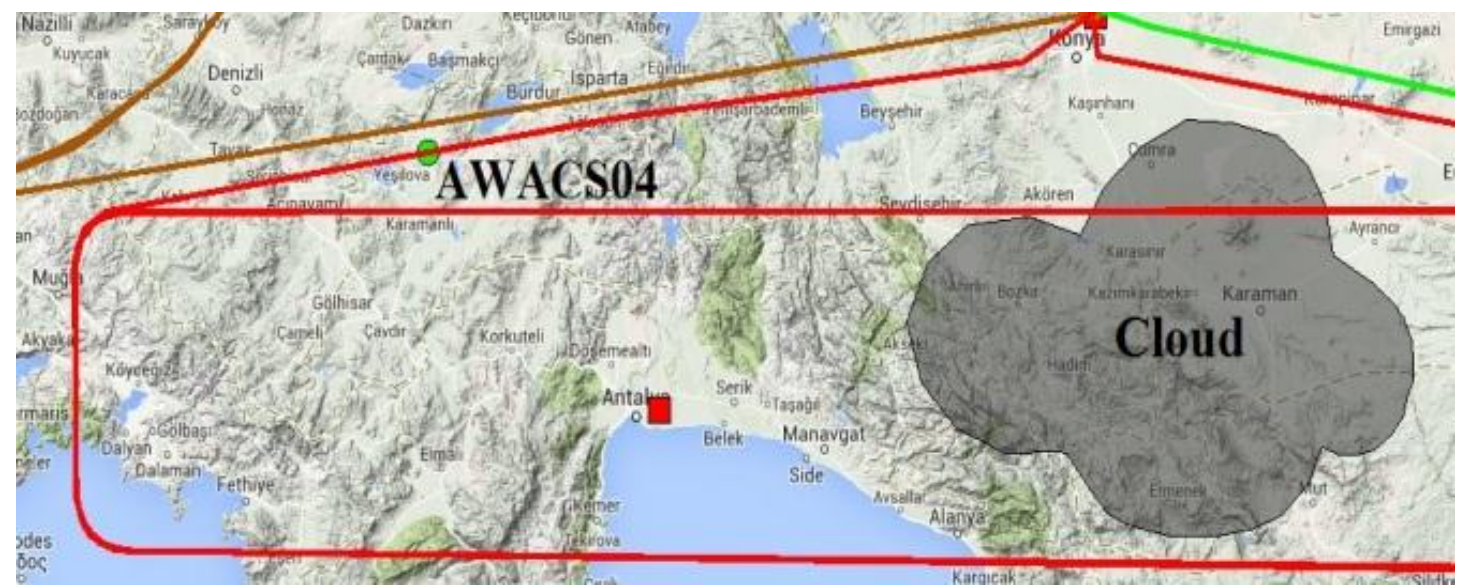

Fig. 1. Point, line and region over Antalya, Turkey.

\subsection{Temporal Databases}

Standard databases normally define the current state of the world. A change in the current state, updates the previous state. For many applications there is a need to keep history. This is possible with standard databases if applications add time values of moving objects manually. So classical data types give very limited support to contain date or time values. Furthermore, working with time values at this form is difficult [4].

Temporal data is formed by time-stamping common data. In a relational data model, relations are extended to have two additional attributes, one for start time and another for end time to establish the time interval of the data [5].

\subsection{Spatio-Temporal Databases}

Spatio-temporal data models deal with geometries move over time [6]. If we wish to describe in the database not only the current state of a spatial data, but also the hole history of a geometry, spatial and temporal data need to be get into interaction. So, continuous changes of geometries mean spatio-temporal data and this is usually expressed as "moving object" [4].

\section{Secondo}

\subsection{Design}

Secondo is one of the first prototype database systems that can demonstrate moving objects, that is, continuously changing time-dependent geometries. More precisely, complete histories of movement can be represented in a database and queried. The basic idea is to use spatio-temporal data types such as moving point and moving region [7].

The goal of Secondo is to form special database system type that consists of various DBMS data models. For example, it should be possible to implement relational, object-oriented, temporal, or XML model. Extensibility to change core data model is special to Secondo [8].

Secondo has a clean architecture. All the source code is accessible, so it is a functional tool for teaching database architecture and implementation concepts [8].

\subsection{Architecture}

Secondo uses Berkeley DB as a storage manager, runs on Windows, Linux, and Mac OS X platforms, and consists of three major components written in different languages [8]:

- The Secondo kernel implements specific data models, is extensible by algebra modules, and provides 
query processing over the implemented algebras. It is implemented on top of Berkeley DB and written in $\mathrm{C}++$ [8].

- The optimizer provides capability of conjunctive query optimization. Conjunctive query optimization is needed for any kind of data models. In addition, it implements the essential part of SQL-like languages. The optimizer is written in PROLOG [8].

- The graphical user interface (GUI) is an extensible interface for new data types or models. There is a specialized viewer available in the GUI for providing a generic and rather sophisticated spatial database interface, including animation of moving objects. The GUI is written in Java [8].

The three components can be used together or independently. All three components can be used together in a configuration shown in Fig. 2 [8].

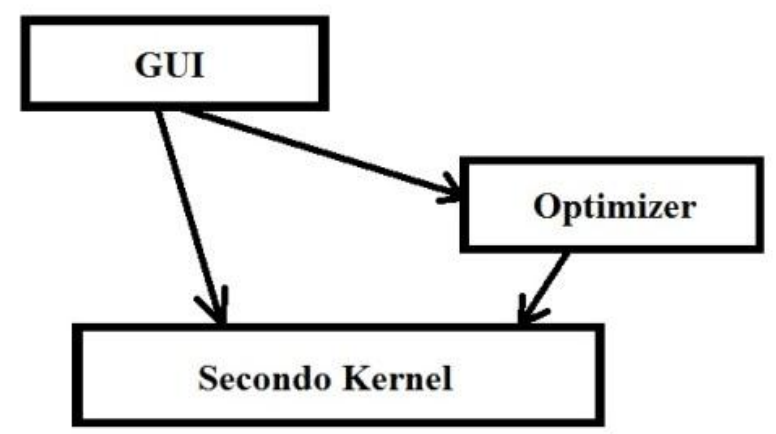

Fig. 2. Cooperation of secondo components.

In this configuration, the GUI can ask the kernel directly to execute commands and queries. Or it can call the optimizer to get a plan for a given SQL query [8].

\subsection{Querying at Secondo}

Secondo is able to run convenient query techniques. Methods are developed to efficiently answer queries such as movement of objects and relations between objects. Most of these queries are not supported by existing methods because of the limitation of data representation [9].

Together with the correct data types, some practical operators for querying events of objects can be used. Some of them are below:

- Trajectory: Computes the route of a moving point.

- Distance: Returns the distance between a moving point and a point.

- Passes: Checks whether a moving point passes through a certain region.

- Inside: Returns the time period when the moving point goes through a moving region [7].

\section{Modeling AWACS in Secondo}

\subsection{System Description}

Peace eagle airborne early warning and control (AEW\&C) aircraft provides advanced airborne surveillance and battle management capabilities and can simultaneously track airborne and maritime targets. With its advanced radar technology it can detect, track and identify small targets, unmanned air vehicles and various missiles to maintain situational awareness and ensure air superiority. With the capability of flying at high altitudes it's able to observe rough territory. Radar technology allows identification of potential targets and friendly aircraft over hundreds of square miles in any direction. Mission duration increases with air to air refueling ability. Thus, AWACS increases the country's self-defense capability. 
The system we designed demonstrates four different AWACS missions and three different types of orbit patterns. Four different mission areas are; east, west, south and north of Turkey. Flight coordinates that we demonstrate are not real.

\subsection{Data Types}

To create moving points; coordinates need to be known. Every point need date and time values. Other values such as altitude, speed, tail number are optional. After data inserted, file must be converted to ".csv" format to be recognized. Data types of moving point values are shown below:

Enlem: real, Boylam: real, Saat: string, Irtifa: real, Hiz: int, Tarih: string, No: int

For moving regions; original coordinates and its starting time and final coordinates and its end time needed. Secondo computes moving region's speed automatically by looking at three values; initial time, end time and the distance between original location and final location. Besides these; for creating point and region, only coordinates needed. Data type of point values are shown below:

$$
\begin{gathered}
\text { OBJECT Bulut() mregion } \\
\text { OBJECT Havaalani () (rel (tuple ( (Name string) (Typ string) (GeoData point)))) } \\
\text { (("Konya" "Havaalan" (32.5608672 37.9786826)) ("Ankara" "Havaalan" (32.8403320 39.9223758)) }
\end{gathered}
$$

\subsection{Operators and Queries}

The system we created let us keep data of objects past movements. Suppose present time is $10^{\text {th }}$ of December 2015, 1 pm. We firstly defined present time. Secondly, we defined forty-eight hours. Here is a query of past event:

$$
\begin{aligned}
& \text { Let presentime=theInstant }(2015,12,10,13,00) \\
& \text { Let fortyeight=[const duration value }(017280000)] \text {; } \\
& \text { Query (Bulut atinstant presentime-fortyeight) }
\end{aligned}
$$

The system lets us observe objects location instantly. We defined present time before. To see the location of these objects queries below have to be executed:

Query (BulutEuro atinstant presentime)

Select [No, val(Rota atinstant presentime) as loc]

From Tumrota where [Rota present presentime]

Analysis of the weather history gives us information about their speed, altitude, direction and size. Every weather object has a characteristic. From this point of view, it's possible to forecast future movement of weather elements.

$$
\begin{aligned}
& \text { Let twentyfour=[const duration value (0 86400000)]; } \\
& \text { Query (Bulut atinstant presentime+twentyeight) }
\end{aligned}
$$

First, twenty-four hours defined. Then twenty-four hours added over present time to observe the location of clouds at $11^{\text {th }}$ of December, 1 pm. Past, present and future location of clouds are shown at Fig. 3. 


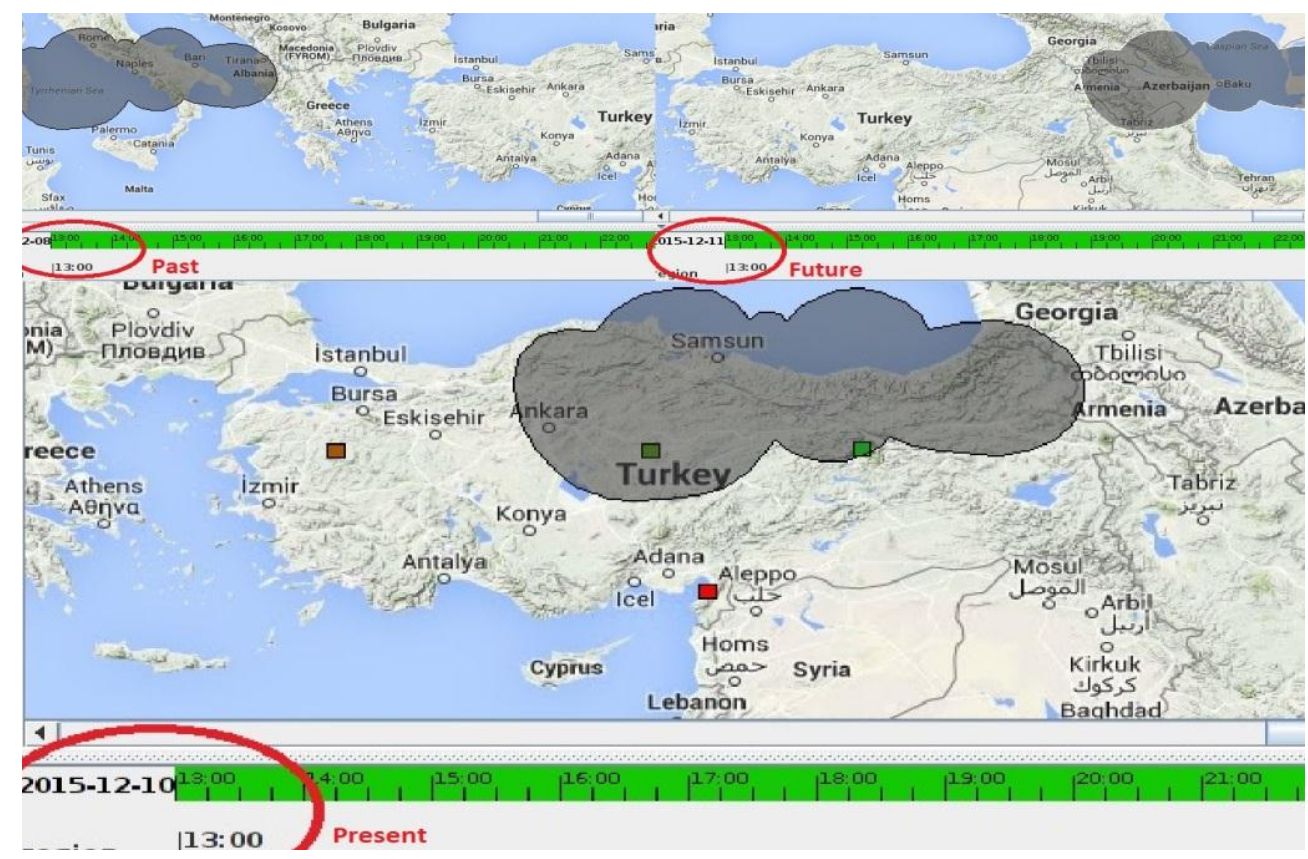

Fig. 3. Past, present and future locations of clouds.

Apart from these, it's possible to achieve queries below:

- What is the location of AWACS aircrafts at nine o'clock?

Let nine $=$ theInstant $(2015,12,10,9,00)$; query Tumrota feed filter[.Rota present nine] consume

- Query that draws the route of each AWACS.

Query Tumrota feed projectextend[No; Traj: trajectory(.Rota)] consume

- Which AWACS aircrafts flight over altitude 40000 feet?

Query Tumrotai feed filter [.Irtifa $>40000$ ] consume

- Which AWACS aircrafts intersect with cloud?

Select [no, intersection (rota, bulut) as bulutici]

From tumrota where [not(isempty(deftime(intersection(rota, bulut))))]

\subsection{Flight Route Modification}

Aviation has come a long way since the Wright brothers first flew the Kitty Hawk. One thing that has unfortunately not changed is the role that weather plays in fatal airplane accidents. Even after a century of flight, weather is still the factor most likely to result in accidents with fatalities [10].

One of the most important activity done about flight is the pre-flight briefing. Pre-flight briefings held before every flight at squadrons. Briefings are compulsory for the flight crew because it helps to be prepared well to the flight. Meteorological condition is an important section which is mentioned at briefings.

Meteorologist mention about sky conditions, icing conditions, cloudiness, visibility, surface winds, lofty winds, precipitation, temperature and Notices to Airmen(NOTAMs). Meteorologists report these issues to flight crew by speaking or just showing the weather chart. With our research weather condition could be demonstrable via GUI. Therefore, pilots could be able to see the movements visually.

So far, Secondo is used to display similar databases for querying only past and present situations. This research will assist future cases to be queried. Past event's data are already known. We could observe cloud's history which arrives eastward from Europe to Turkey. Its data such as coordinates, shape, size, directions, speed, altitude etc. recorded instantly. Thus, analyzing historic data can help us forecast when and where clouds may be in the future. Fig. 4 shows timeline of weather events. 


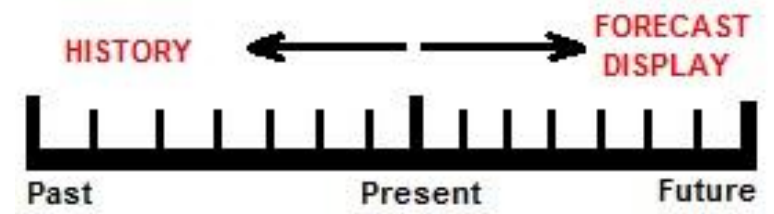

Fig. 4. Timeline of weather events.

Meteorologists, who come to squadrons early in the morning, to give briefing, just need to enter the current weather condition. Secondo will compute and demonstrate the estimated location of clouds.

AWACS's missions, routes and planned take-off, landing times are certain. If meteorologists point out the forecast of weather chart and show where and when the clouds going to be, flight crew can take precautions before take-off. Showing the movement of weather entities and planes visually is highly beneficial. At Fig. 5, we queried intersection of AWACSs and cloud. To see if AWACS aircrafts encounter with hazardous weather conditions, several queries can be used. In this way, flight route can be modified, planned mission can be canceled and take-off time can be changed to maintain safety.

\section{Select * From Ucus \\ Where[not(isempty(deftime(intersection(Rota,Bulut))))]}

Another important flight danger is volcanic ash clouds. Volcanic ash is pulverized rock. If these small particles go into the jet engines, they can significantly affect the performance of jet engines at cruise altitudes. Ash clouds are often invisible, particularly at night [11]. Thus, Secondo can also be convenient to solve this problem. A research can be done to follow up the volcanic ash cloud's movement.

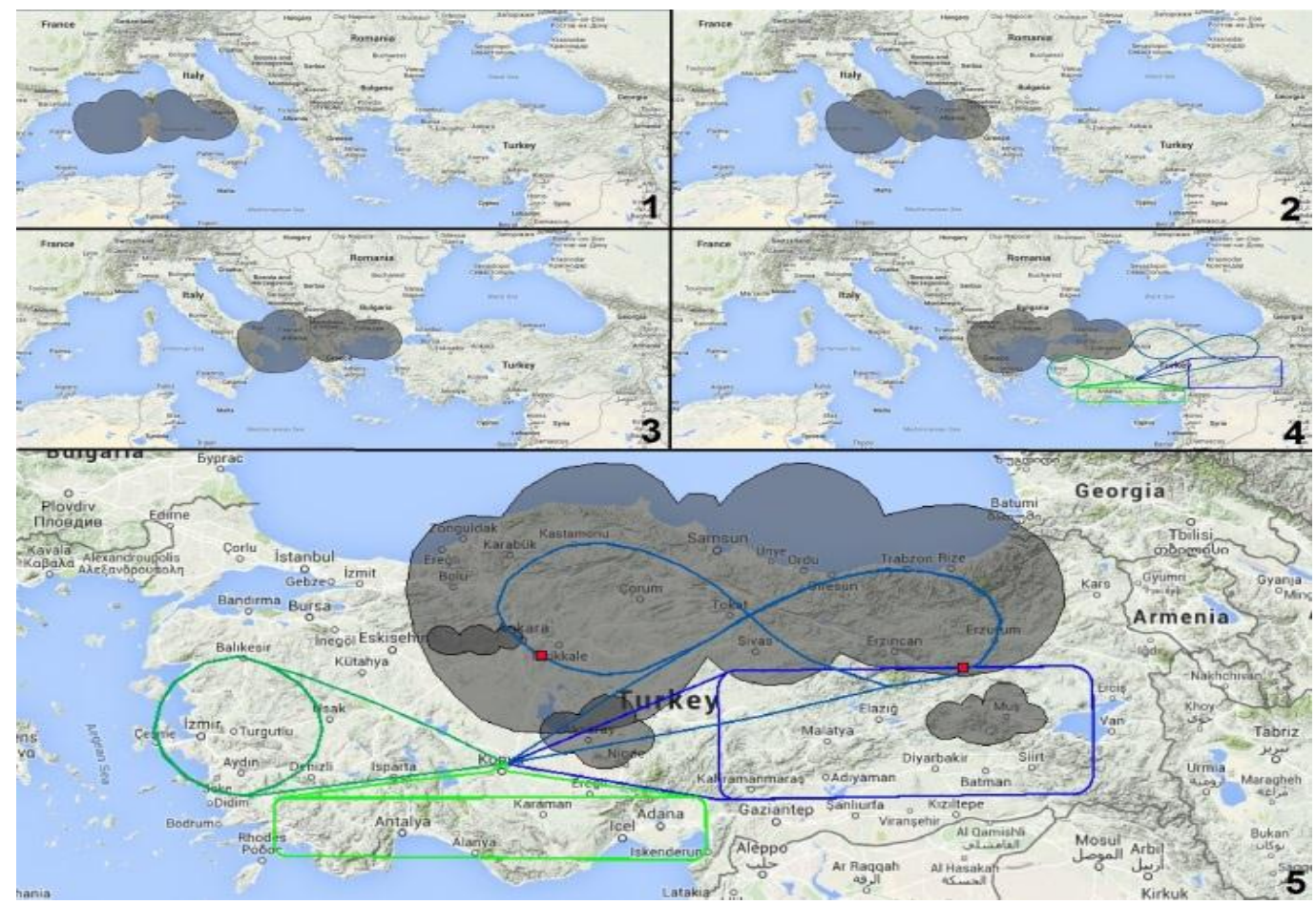

Fig. 5. Intersection of AWACSs and cloud.

\section{Conclusion}

We explained spatio-temporal databases and mentioned differences between classical databases. Moving object databases are rapidly developing field and have a wide range of usage. 
Using Secondo at AWACS route modification according to weather forecasting can make AWACS squadron more useful. Flights may become safer. AWACS squadron's development at visual forecasting may help Turkish Armed Forces, double its power and increase its defense.

Besides, this idea can be used all around the World at commercial aircrafts with integrating radar scope to the cockpit which holds instantly updatable weather conditions. This may make airlines safer.

\section{References}

[1] Li, Z., Ji, M., Lee, J.-G., Tang, L.-A., Yu, Y., Han, J., \& Kays, R. (2010). Movemine: Mining moving object databases. Proceedings of SIGMOD'10.

[2] Damiani, M. L., Issa, H., Guting, R. H., \& Valdes, F. (July 2014). Hybrid queries over symbolic and spatial trajectories: A usage scenario. Proceedings of 2014 IEEE 15th International Conference on Mobile Data Management (pp. 341-344).

[3] Ayabakan, İ., \& Kilimci, P. (June 2014). Hareketli Nesne Veri Tabanları İndeksleme Algoritmaları ve En Yakın Komşuluk Yaklaşımı. Hava Harp Okulu-Havacılık ve Uzay Teknolojileri Enstitüsü.

[4] Guting, R. H., \& Schneider, M. (2005). Moving Object Databases. Morgan Koufmann.

[5] Patel, J. (2003). Temporal Database Systems. University of London.

[6] Chen, C. X. (2001). Data models and query languages of spatio-temporal information. Univ. of California.

[7] Secondo Team. (2011). A Database System for Moving Objects.

[8] Guting, R. H., Ansorge, D., Behr, T., Düntgen, C., Jandt, S., \& Spiekermann, M. (July 2011). Secondo User Manuel-Database Systems for New Applications. Fern University, Hagen.

[9] Xu, J., \& Guting, R. H. (2012). Manage and Query Generic Moving Objects in SECONDO. Fern University.

[10] Parson, S. (2009). General Aviation Pilot's Guide to Preflight Weather Planning, Weather Self-Briefings, and Weather Decision Making. FAA.

[11] Kulesa, G. (2002). Weather and Aviation: How Does Weather Affect the Safety and Operations of Airports and Aviation, and How Does FAA Work to Manage Weather-related Effects?

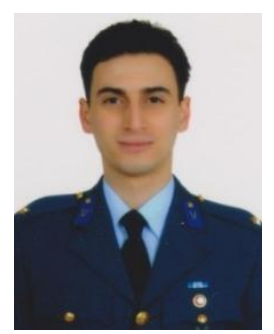

Konuralp Küçüködük was born in Istanbul, Turkey, in 1987. He received the bachelor's degree in computer engineering from Turkish Air Force Academy, İstanbul, Turkey, in 2009 and he joined the Turkish Air Force as a lieutenant.

Konuralp finished the flight training at the Flight School, Izmir, Turkey, in 2010. In 2011, he received the training of signal officer at the Air Force Technical School, İzmir, Turkey. In 2011, he was appointed to Konya, Turkey as a software developer at AEW\&C (Airborne Early Warning and Control). In 2012, he became a first lieutenant. Since 2013, he has been educating as a master student at Turkish Air Force Academy Aeronautics and Space Technologies Institute, Istanbul, Turkey.

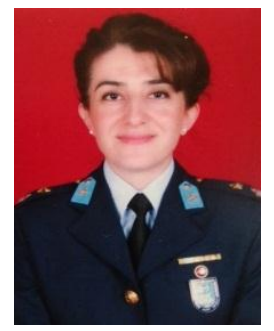

Perihan Pehlivanoğlu graduated from the Computer Engineering Department at Ege University in 1995. She got her Ph.D. degree in 2009. Currently, she teaches databases and object oriented programming courses at Noncommissioned Air Officer College. 